\title{
Identification of biomarkers and candidate inhibitors for multiple myeloma
}

Hanming Gu ${ }^{1,2}$, Wei Wang ${ }^{3}$, Gongsheng Yuan ${ }^{4}$

${ }^{1}$ School of Electronic, Information and Electrical Engineering, Shanghai Jiao Tong University, Shanghai, China

${ }^{2}$ SHU-UTS SILC Business School, Shanghai University, Shanghai, China

${ }^{3}$ Shanghai Civil Aviation College, Shanghai, China

${ }^{4}$ Department of Physiology and Pathophysiology, School of Basic Medical Sciences, Fudan University, Shanghai, China

"Corresponding author: Dr. Gongsheng Yuan, Department of Physiology and Pathophysiology, School of Basic Medical Sciences, Fudan University, Shanghai, China gsyuan14@gmail.com 


\section{Abstract}

Multiple myeloma (MM) is a plasma cell malignancy that causes the overabundance of monoclonal paraprotein (M protein) and organ damages. In our study, we aim to identify biological markers and processes of MM using a bioinformatics method to elucidate their potential pathogenesis. The gene expression profiles of the GSE153626 datasets were originally produced by using the high-throughput Illumina HiSeq 4000 (Mus musculus). The functional categories and biochemical pathways were identified and analyzed by the Kyoto Encyclopedia of Genes and Genomes pathway (KEGG), Gene Ontology (GO), and Reactom enrichment. KEGG and GO results showed the biological pathways related to immune dysfunction and signal transduction are mostly affected in the development of MM. Moreover, we identified several genes including Gngt2, Foxp3, and $\mathrm{Cd} 3 \mathrm{~g}$ were involved in the regulation of immune cells. We further predicted new inhibitors that have the ability to block the progression of MM based on the L1000fwd analysis. Therefore, this study provides further insights into the underlying pathogenesis of MM.

\section{Introduction}

Multiple myeloma is hematological cancer that caused end-organ damage including anemia, renal impairment, and bone lesions ${ }^{1}$. MM primarily occurs in the elderly (over 65 years) and the global incidence has increased dramatically since $1990^{2,3}$. With current chemotherapy and regimens, the survival rate has improved, although it is still a high-risk disease ${ }^{4}$. Malignant migration of MM is a consequence of a combination of factors such as genetic mutations and genetic evolutions in the BM environment ${ }^{5}$. Moreover, immune dysfunction was found in myeloma patients, raising the question of whether immunological dysregulation is an important mechanism of disease development $^{6,7}$.

Most of the findings showed that several types of mouse models have been used, which aim to provide insights on the relationship between MM and BM microenvironment ${ }^{8}$. Here, the mouse model we analyzed was created through conditionally activating the endogenous NrasQ61R and an MYC transgene in germinal center B cells ${ }^{9}$. This mouse model developed a classic malignant MM characterized by phenotype, signaling 
pathways, and MM gene signatures ${ }^{9}$. Recent studies suggest that the uncontrolled immunological microenvironment leads to the progress of $\mathrm{MM}^{10}$. The activated MM may cause bone damage, anemia, multiple organ metastasis ${ }^{11}$. However, the mechanism underlying MM is still unknown.

Here, we studied the relative changes of genes in MM mouse models. Then, we identified and analyzed a series of DEGs, the relevant biological process, and potential drug targets of MM using comprehensive bioinformatic analysis. Last, we performed the gene and pathway analysis, the functional enrichment, and protein-protein interaction (PPI) for discovering MM gene nodes. The critical genes and pathways could be in favor of future clinical and therapeutic studies.

\section{Methods}

Data resources

The dataset GSE153626 was downloaded from the GEO database (http://www.ncbi.nlm.nih.gov/geo/). The data was produced by Illumina HiSeq 4000 (Mus musculus), Blood Research Institute, Versiti 8727 W Watertown Plank Rd, Milwaukee, Wisconsin. Bulk RNA-Seq analysis was performed using CD138+ B220CD45.2+ bone marrow cells of three control and five MM models conditionally activating expression of endogenous NrasQ61R and a MYC transgene in germinal center B cells (VQ mice).

Data acquisition and preprocessing

The dataset GSE153626 containing WT samples and VQ mice samples was analyzed and conducted by $R$ script as described previously ${ }^{12,13}$. We used a classical t test to identify $D E G$ with $P<.01$ and fold change $\geq 1.5$ as being statistically significant.

Gene functional analysis

Gene ontology (GO) analysis is a widely used approach to develop a comprehensive and computational model of biological systems to analyze genomic data and define characteristic biological information $^{14}$. The Kyoto Encyclopedia of Genes and Genomes (KEGG) database is commonly used for understanding high-level functions and utilities 
of the biological system ${ }^{15}$. We performed the GO analysis and KEGG pathway enrichment analysis by using the Database for Annotation, Visualization, and Integrated Discovery (DAVID) (http://david.ncifcrf.gov/) as described previously ${ }^{16,17} . \mathrm{P}<.05$ and gene counts $>10$ were considered statistically significant.

Module analysis

The Molecular Complex Detection (MCODE) of cytoscape software was used to analyze the densely connected regions in protein-protein interaction (PPI) networks ${ }^{18}$. The significant modules were from the constructed PPI network using MCODE ${ }^{19}$. The function and pathway enrichment analyses were performed by using DAVID, and $P<.05$ was used as the cutoff criterion.

Reactome pathway analysis

The dataset was performed by the Reactom pathway (https://reactome.org/) to obtain the visualization, interpretation and analysis of potential pathways. $\mathrm{P}<.05$ was considered statistically significant.

\section{Results}

\section{Identification of DEGs in myeloma cells from MM models}

Multiple myeloma mouse models were created by activating the expression of endogenous NrasQ61R and a MYC transgene in germinal center B cells (VQ mice) at Blood Research Institute, Versiti (Wisconsin, USA). To gain the insights on MM model genes, the modular transcriptional signature of VQ mice was compared to that of WT mice. A total of 3721 genes were identified to be differentially expressed in MM models with the threshold of $\mathrm{P}<0.005$. The top 10 up- and down-regulated genes for $\mathrm{VQ}$ mice and WT control samples are listed in Table 1.

\section{KEGG analysis of DEGs in MM models}

To further identify the biological roles and potential mechanisms of the DEGs from the MM models versus WT controls, we performed KEGG pathway and GO categories enrichment analysis (Supplemental Table S1). KEGG pathways 
(http://www.genome.jp/kegg/) are a collection of manually drawn pathway maps for understanding the molecular interaction, reaction and relation networks. Our study presented the top ten enriched KEGG pathways including "Inflammatory bowel disease (IBD)", "Hematopoietic cell lineage", "African trypanosomiasis", "Transcriptional misregulation in cancer", "Pancreatic secretion", "Fc epsilon RI signaling pathway", "T cell receptor signaling pathway", "Natural killer cell mediated cytotoxicity", "Salivary secretion", and "Oxytocin signaling pathway" (Figure 1).

\section{GO analysis of DEGs in MM models}

Gene ontology (GO) analysis is a system for hierarchically classifying genes, which includes cellular components (CC), molecular functions (MF), and biological processes $(\mathrm{BP})^{20}$. Here, we identified top ten cellular components including "Membrane", "External side of plasma membrane", "Cell surface", "Plasma membrane", "Cell-cell junction", "Mast cell granule", "Extracellular exosome", "Cytoplasmic side of plasma membrane", "Early endosome", and "Integral component of membrane" (Figure 1). We then identified top ten biological processes: "Negative regulation of interleukin-10 production", "Positive regulation of Rho protein signal transduction", "Myeloid cell differentiation", "Negative regulation of T cell proliferation", "B cell activation", "Positive regulation of T cell proliferation", "Immune system process", “Adaptive immune response”, "Immune response", and "Positive regulation of gene expression" (Figure 1). Last, we identified top ten molecular functions: "Protein binding", "Receptor activity", "Guanyl-nucleotide exchange factor activity", "Cysteine-type endopeptidase inhibitor activity involved in apoptotic process", "ATPase binding", "Semaphorin receptor activity", "Transcription regulatory region DNA binding", "Phosphoprotein phosphatase activity", "Actin binding", and "Signal transducer activity" (Figure 1).

\section{PPI network and Module analysis}

The PPI networks were created to analyze the relationships of DGEs at the protein level $^{21,22}$. The criterion of combined score $>0.7$ was chosen and the PPI network was constructed by using the 143 nodes and 209 interactions. Among these nodes, the top ten genes with highest scores are shown in Table 2. The top two significant modules of 
MM models versus WT samples were selected to indicate the functional annotation (Figure 2).

\section{Reactome Pathway in MM models}

We identified several signaling pathways by using Reactome Pathway Database (https://reactome.org/). We identified top ten signaling pathways including: "Interleukin35 Signalling", "RUNX1 and FOXP3 control the development of regulatory T lymphocytes (Tregs)", "Other semaphorin interactions", "TFAP2 (AP-2) family regulates transcription of growth factors and their receptors", "Interleukin-18 signaling", "Neurophilin interactions with VEGF and VEGFR", "Defective factor IX causes hemophilia B", "Interleukin-12 family signaling", "Interleukin-38 signaling", and "RUNX1 regulates transcription of genes involved in BCR signaling" (Supplemental Table S2). We then constructed the reaction map according to the signaling pathways (Figure 3).

\section{Potential inhibitors in MM models}

To further know how to prevent the MM, we introduced the L1000FDW system that can predict and analyze the potential inhibitors (Figure 4). With the mechanisms-of-action (MOA), the L1000FDW system indicated the most potentially affected pathways. We selected top ten inhibitors according to the DEGs and the inhibitor maps: "BRDA47816767", "radicicol", "melperone", "IKK-2-inhibitor-V", "I-606051", "alitretinoin", "BRD-K70771662", "SSR-69071", "SA-1447005”, and "CAY-10470" (Supplemental Table S3).

\section{Discussion}

$\mathrm{MM}$ is a plasma cell malignancy that accounts for about $10 \%$ of hematologic cancers ${ }^{23}$. MM causes several symptoms such as organ damage, anemia and lytic bony lesions ${ }^{24}$. MM was triggered from a premalignant state called "monoclonal gammopathy of undetermined significance (MGUS)" ${ }^{25}$. Malignant transformation is the result of combinations of multiple factors such as genes and BM microenvironment ${ }^{26}$. Moreover, immune dysfunction plays an important role in the progression of $\mathrm{MM}^{6}$. Thus, the signature of genes and proteins are the key targets to prevent the diseases. 
To better understand the effects of inhibitors on the MM mouse models, we analyzed a novel MM mouse model that was created by Blood Research Institute, Versiti. This model was based on the conditionally activating expression of endogenous NrasQ61R and a MYC transgene in germinal center $B$ cells $(V Q \text { mice })^{9}$. Thus, by using this transgenic mouse we could produce more MM models to test the drugs and inhibitors as previously described ${ }^{17}$. By analyzing the DEGs of this model, we selected 10 proteins that may be important during the development of MM according to the PPI network analysis. G proteins and RGS proteins are widely involved in the immune and inflammatory diseases. In our study, the Guanine nucleotide-binding protein $\mathrm{G}(\mathrm{I}) / \mathrm{G}(\mathrm{S}) / \mathrm{G}(\mathrm{O})$ subunit gamma-T2 is a potential prognostic marker of esophageal cancer $^{27}$. The FOXP3 in T-cells can suppress antigen priming of lymphocytes and can be regulated by the multifunctional circadian clocks ${ }^{28-30}$. CD3g deletion from a consanguineous family is associated with autoimmunity ${ }^{31}$. H2-Ab1 and H2-Eb2 are reported to be involved in the control of tuberculosis infection ${ }^{32}$. G-protein-coupled receptor (GPCR) proteins and RGS proteins are highly related to the tumor genesis ${ }^{33-36}$. F2RI2 is recently reported to be a GPCR that encodes protease-activated receptor-3 $(\mathrm{PAR} 3)^{37}$. Circadian clock controls numerous genes to regulate the cell functions during normal conditions and diseases ${ }^{29,38-41}$. As a clock-controlled gene, CCR5 is reported to suppress anti-tumor immune response and promote tumor growth ${ }^{42,43}$. Long and short isoforms of CEACAM1 are strongly expressed on cancer cells in the liver metastases ${ }^{44}$. VAV3 is associated with the ERBB4-mediated cancer cell migration ${ }^{45}$. LAT-1 is identified as a promotor in gastric cancer that is related to the clinicopathologic features ${ }^{46}$.

KEGG and GO analysis showed that immune responses play a critical role in the progression of MM. The KEGG analysis showed the "Natural killer cell mediated cytotoxicity", "T cell receptor signaling pathway", "Fc epsilon RI signaling pathway" and "Inflammatory bowel disease (IBD)" were the major pathways during the development of $\mathrm{MM}$. It is suggested that the occurrence of MM is based on the inflammation or immune changes. Similarly, MM is reported to be caused by the disrupted immune surveillance such as deregulation of the $T$ and natural killer cell compartment and recruitment of immunosuppressive cells ${ }^{47}$. Interestingly, the BP of GO analysis showed "Negative 
regulation of IL10 production", "Negative regulation of T cell proliferation" and "B cell activation", suggesting that the multiple immune cells were involved in the progression of MM not only B cells. Thus, the BM microenvironmental condition is crucial in MM. Indeed, the MM cells disseminate in the BM microenvironment via interactions of the adhesion molecules and extracellular matrix (ECM) components and receive various signals that maintain their survival and activity ${ }^{48}$.

Despite recent advancements in drug development, the anti-cancer drug resistance is a major limitation of MM therapy. Thus, the potential inhibitors may be a good substitute for the traditional drugs of MM. In our study, we predicted several inhibitors of MM that most of them are involved in the regulation of immune and protein synthesis pathways such as NF-KB pathways. NF-KB pathway is a center of inflammation which is widely associated in the regulations and controls of diseases ${ }^{36,49}$. However, the side effect of NF-KB inhibitors is so strong that they cannot be used in clinical ${ }^{50}$. Here, besides NF-KB inhibitors, we selected several novel inhibitors that may take effects on the MM mouse models. Our future studies will mainly target the effects on the treatment of MM by using MM mouse models or primary MM cells.

In summary, we identified the potential biomarkers for MM. Immune dysfunction and BM microenvironment are two key processes in the novel MM mouse models. Future studies will focus on the administration of potential inhibitors on clinical trials. This study thus provides further insights into the mechanism of MM, which may facilitate the diagnosis and drug development. 


\section{Reference}

[1] Al-Farsi K: Multiple myeloma: an update. Oman Med J 2013, 28:3-11.

[2] Rajkumar SV: Multiple myeloma. Curr Probl Cancer 2009, 33:7-64.

[3] Hussain A, Almenfi HF, Almehdewi AM, Hamza MS, Bhat MS, Vijayashankar NP: Laboratory Features of Newly Diagnosed Multiple Myeloma Patients. Cureus 2019, 11:e4716.

[4] Multiple myeloma: 2018 update on diagnosis, risk-stratification, and management. Am J Hematol 2018, 93:981-1114.

[5] Chin L, Garraway LA, Fisher DE: Malignant melanoma: genetics and therapeutics in the genomic era. Genes Dev 2006, 20:2149-82.

[6] Romano A, Conticello C, Cavalli M, Vetro C, La Fauci A, Parrinello NL, Di Raimondo F: Immunological dysregulation in multiple myeloma microenvironment. Biomed Res Int 2014, 2014:198539.

[7] Soekojo CY, Ooi M, de Mel S, Chng WJ: Immunotherapy in Multiple Myeloma. Cells 2020, 9.

[8] Rossi M, Botta C, Arbitrio M, Grembiale RD, Tagliaferri P, Tassone P: Mouse models of multiple myeloma: technologic platforms and perspectives. Oncotarget 2018, 9:20119-33.

[9] Wen Z, Rajagopalan A, Flietner ED, Yun G, Chesi M, Furumo Q, Burns RT, Papadas A, Ranheim EA, Pagenkopf AC, Morrow ZT, Finn R, Zhou Y, Li S, You X, Jensen J, Yu M, Cicala A, Menting J, Mitsiades CS, Callander NS, Bergsagel PL, Wang D, Asimakopoulos F, Zhang J: Expression of NrasQ61R and MYC transgene in germinal center B cells induces a highly malignant multiple myeloma in mice. Blood 2021, 137:61-74.

[10] Fairfield H, Falank C, Avery L, Reagan MR: Multiple myeloma in the marrow: pathogenesis and treatments. Ann N Y Acad Sci 2016, 1364:32-51.

[11] Ghobrial IM: Myeloma as a model for the process of metastasis: implications for therapy. Blood 2012, 120:20-30.

[12] Gu H, Yuan G: Identification of key genes in SARS-CoV-2 patients on bioinformatics analysis. bioRxiv 2020:2020.08.09.243444.

[13] Hanming G, Wei W, Gongsheng Y: Research Square 2020.

[14] Pomaznoy M, Ha B, Peters B: GOnet: a tool for interactive Gene Ontology analysis. BMC Bioinformatics 2018, 19:470.

[15] Kanehisa M, Goto S: KEGG: kyoto encyclopedia of genes and genomes. Nucleic Acids Res 2000, 28:27-30.

[16] Gu H, Yuan G: Identification of potential key genes for SARS-CoV-2 infected human bronchial organoids based on bioinformatics analysis. bioRxiv 2020:2020.08.18.256735.

[17] Gu H, Yuan G: Identification of potential biomarkers and inhibitors for SARS-CoV-2 infection. medRxiv 2020:2020.09.15.20195487.

[18] Li M, Li D, Tang Y, Wu F, Wang J: CytoCluster: A Cytoscape Plugin for Cluster Analysis and Visualization of Biological Networks. Int J Mol Sci 2017, 18.

[19] Gu H, Yuan G: Identification of specific biomarkers and pathways in the synovial tissues of patients with osteoarthritis in comparison to rheumatoid arthritis. bioRxiv 2020:2020.10.22.340232.

[20] Roncaglia P, Martone ME, Hill DP, Berardini TZ, Foulger RE, Imam FT, Drabkin H, Mungall CJ, Lomax J: The Gene Ontology (GO) Cellular Component Ontology: integration with SAO (Subcellular Anatomy Ontology) and other recent developments. J Biomed Semantics 2013, 4:20.

[21] Safari-Alighiarloo N, Taghizadeh M, Rezaei-Tavirani M, Goliaei B, Peyvandi AA: Protein-protein interaction networks (PPI) and complex diseases. Gastroenterol Hepatol Bed Bench 2014, 7:17-31.

[22] Hanming G, Gongsheng Y: Research Square 2021.

[23] Rajkumar SV, Kumar S: Multiple Myeloma: Diagnosis and Treatment. Mayo Clin Proc 2016, 91:10119. 
[24] Hameed A, Brady JJ, Dowling P, Clynes M, O'Gorman P: Bone disease in multiple myeloma: pathophysiology and management. Cancer Growth Metastasis 2014, 7:33-42.

[25] Zingone A, Kuehl WM: Pathogenesis of monoclonal gammopathy of undetermined significance and progression to multiple myeloma. Semin Hematol 2011, 48:4-12.

[26] Korn C, Mendez-Ferrer S: Myeloid malignancies and the microenvironment. Blood 2017, 129:811-22.

[27] Liu GM, Ji X, Lu TC, Duan LW, Jia WY, Liu Y, Sun ML, Luo YG: Comprehensive multi-omics analysis identified core molecular processes in esophageal cancer and revealed GNGT2 as a potential prognostic marker. World J Gastroenterol 2019, 25:6890-901.

[28] Yang G, Zhang H, Liu Y, Feng Y, Luo XQ, Liu ZQ, Geng XR, Wang S, Zheng PY, Feng BS, Liu ZG, Yang PC, Li HB, Wu SD: Alternation of circadian clock modulates forkhead box protein-3 gene transcription in CD4(+) T cells in the intestine. J Allergy Clin Immunol 2016, 138:1446-9 e10.

[29] Yuan G, Xu L, Cai T, Hua B, Sun N, Yan Z, Lu C, Qian R: Clock mutant promotes osteoarthritis by inhibiting the acetylation of NFkappaB. Osteoarthritis Cartilage 2019, 27:922-31.

[30] Yuan G, Hua B, Cai T, Xu L, Li E, Huang Y, Sun N, Yan Z, Lu C, Qian R: Clock mediates liver senescence by controlling ER stress. Aging 2017, 9:2647-65.

[31] Gokturk B, Keles S, Kirac M, Artac H, Tokgoz H, Guner SN, Caliskan U, Caliskaner Z, van der Burg M, van Dongen J, Morgan NV, Reisli I: CD3G gene defects in familial autoimmune thyroiditis. Scand J Immunol 2014, 80:354-61.

[32] Logunova N, Korotetskaya M, Polshakov V, Apt A: The QTL within the H2 Complex Involved in the Control of Tuberculosis Infection in Mice Is the Classical Class II H2-Ab1 Gene. PLoS Genet 2015, 11:e1005672.

[33] Fu C, Yuan G, Yang ST, Zhang D, Yang S: RGS12 Represses Oral Cancer via the Phosphorylation and SUMOylation of PTEN. J Dent Res 2020:22034520972095.

[34] Kimple AJ, Bosch DE, Giguere PM, Siderovski DP: Regulators of G-protein signaling and their Galpha substrates: promises and challenges in their use as drug discovery targets. Pharmacol Rev 2011, 63:72849.

[35] Yuan G, Yang S, Liu M, Yang S: RGS12 is required for the maintenance of mitochondrial function during skeletal development. Cell Discov 2020, 6:59.

[36] Yuan G, Yang S, Gautam M, Luo W, Yang S: Macrophage regulator of G-protein signaling 12 contributes to inflammatory pain hypersensitivity. Annals of Translational Medicine 2021.

[37] Hanzelmann S, Wang J, Guney E, Tang Y, Zhang E, Axelsson AS, Nenonen H, Salehi AS, Wollheim CB, Zetterberg E, Berntorp E, Costa IG, Castelo R, Rosengren AH: Thrombin stimulates insulin secretion via protease-activated receptor-3. Islets 2015, 7:e1118195.

[38] Yuan G, Hua B, Yang Y, Xu L, Cai T, Sun N, Yan Z, Lu C, Qian R: The Circadian Gene Clock Regulates Bone Formation Via PDIA3. J Bone Miner Res 2017, 32:861-71.

[39] Zhu Z, Hua B, Shang Z, Yuan G, Xu L, Li E, Li X, Sun N, Yan Z, Qian R, Lu C: Altered Clock and Lipid Metabolism-Related Genes in Atherosclerotic Mice Kept with Abnormal Lighting Condition. Biomed Res Int 2016, 2016:5438589.

[40] Cai T, Hua B, Luo D, Xu L, Cheng Q, Yuan G, Yan Z, Sun N, Hua L, Lu C: The circadian protein CLOCK regulates cell metabolism via the mitochondrial carrier SLC25A10. Biochim Biophys Acta Mol Cell Res 2019, 1866:1310-21.

[41] Zhu Z, Hua B, Xu L, Yuan G, Li E, Li X, Sun N, Yan Z, Lu C, Qian R: CLOCK promotes 3T3-L1 cell proliferation via Wnt signaling. IUBMB Life 2016, 68:557-68.

[42] Li BH, Garstka MA, Li ZF: Chemokines and their receptors promoting the recruitment of myeloidderived suppressor cells into the tumor. Mol Immunol 2020, 117:201-15.

[43] Chen X, Hu Q, Zhang K, Teng H, Li M, Li D, Wang J, Du Q, Zhao M: The clock-controlled chemokine contributes to neuroinflammation-induced depression. FASEB J 2020, 34:8357-66. 
[44] Yamaguchi S, Yokoyama S, Ueno M, Hayami S, Mitani Y, Takeuchi A, Shively JE, Yamaue H: CEACAM1 is associated with recurrence after hepatectomy for colorectal liver metastasis. J Surg Res 2017, 220:353-62.

[45] Ojala VK, Knittle AM, Kirjalainen P, Merilahti JAM, Kortesoja M, Tvorogov D, Vaparanta K, Lin S, Kast J, Pulliainen AT, Kurppa KJ, Elenius K: The guanine nucleotide exchange factor VAV3 participates in ERBB4-mediated cancer cell migration. J Biol Chem 2020, 295:11559-71.

[46] Wang J, Chen X, Su L, Li P, Liu B, Zhu Z: LAT-1 functions as a promotor in gastric cancer associated with clinicopathologic features. Biomed Pharmacother 2013, 67:693-9.

[47] Rodriguez-Otero P, Paiva B, Engelhardt M, Prosper F, San Miguel JF: Is immunotherapy here to stay in multiple myeloma? Haematologica 2017, 102:423-32.

[48] Robak P, Drozdz I, Szemraj J, Robak T: Drug resistance in multiple myeloma. Cancer Treat Rev 2018, 70:199-208.

[49] Yuan G, Yang S, Ng A, Fu C, Oursler MJ, Xing L, Yang S: RGS12 Is a Novel Critical NF-kappaB Activator in Inflammatory Arthritis. iScience 2020, 23:101172.

[50] Ramadass V, Vaiyapuri T, Tergaonkar V: Small Molecule NF-kappaB Pathway Inhibitors in Clinic. Int J Mol Sci 2020, 21. 
bioRxiv preprint doi: https://doi.org/10.1101/2021.02.25.432847; this version posted February 25, 2021. The copyright holder for this preprint (which was not certified by peer review) is the author/funder, who has granted bioRxiv a license to display the preprint in perpetuity. It is made available under aCC-BY-NC-ND 4.0 International license.

KEGG pathways

Inflammatory bowel disease (IBD)

African trypanosomiasis Hematopoietic cell lineage

Fc epsilon RI signaling pathway

Salivary secretion

Pancreatic secretion

T cell receptor signaling pathway

Natural killer cell mediated cytotoxicity
Transcriptional misregulation in cancer Oxytocin signaling pathway

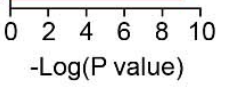

Cellular component

Integral component of membrane

Plasma membrane

Extracellular exosome

Membrane

Early endosome

Cell surface

Cell-cell junction

External side of plasma membrane

Cytoplasmic side of plasma membrane

Mast cell granule

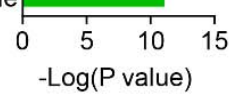

Biological Process

Positive regulation of gene expression

Immune response

Adaptive immune response

Immune system process

Positive regulation of $\mathrm{T}$ cell proliferation

$B$ cell activation

Negative regulation of $\mathrm{T}$ cell proliferation

Myeloid cell differentiation

Positive regulation of Rho protein signal transduction Negative regulation of interleukin-10 production

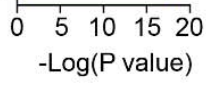

Molecular Function

Protein binding Signal transducer activity Actin binding

Transcription regulatory region DNA binding Phosphoprotein phosphatase activity Guanyl-nucleotide exchange factor activity Receptor activity

Cysteine-type endopeptidase inhibitor activity Semaphorin receptor activity

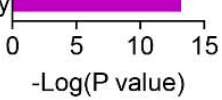

Figure 1. The KEGG pathways, biological process, cellular component, and molecular function terms enriched by the DEGs between WT and MM mouse models. $\mathrm{DEGs}=$ differentially expressed genes, KEGG $=$ Kyoto Encyclopedia of Genes and Genomes. 
bioRxiv preprint dol: https://doi.org/10.1101/2021.02.25.432847; this version posted February $25,2021$. The copyright holder for this preprint

(which was not certified by peer review) is the author/funder, who has granted bioRxiv a license to display the preprint in perpetuity. It is made available under aCC-BY-NC-ND 4.0 International license.
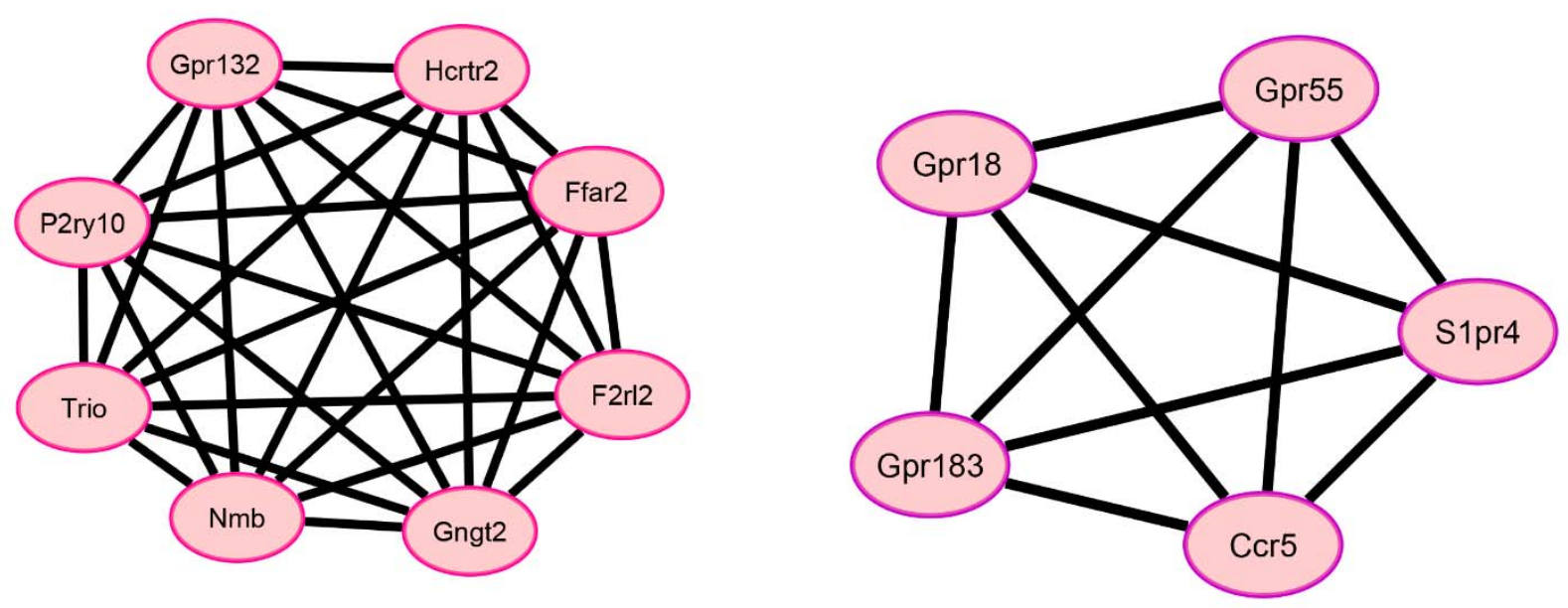

Figure 2. Top two modules from the PPI network between WT and MM mouse models. 


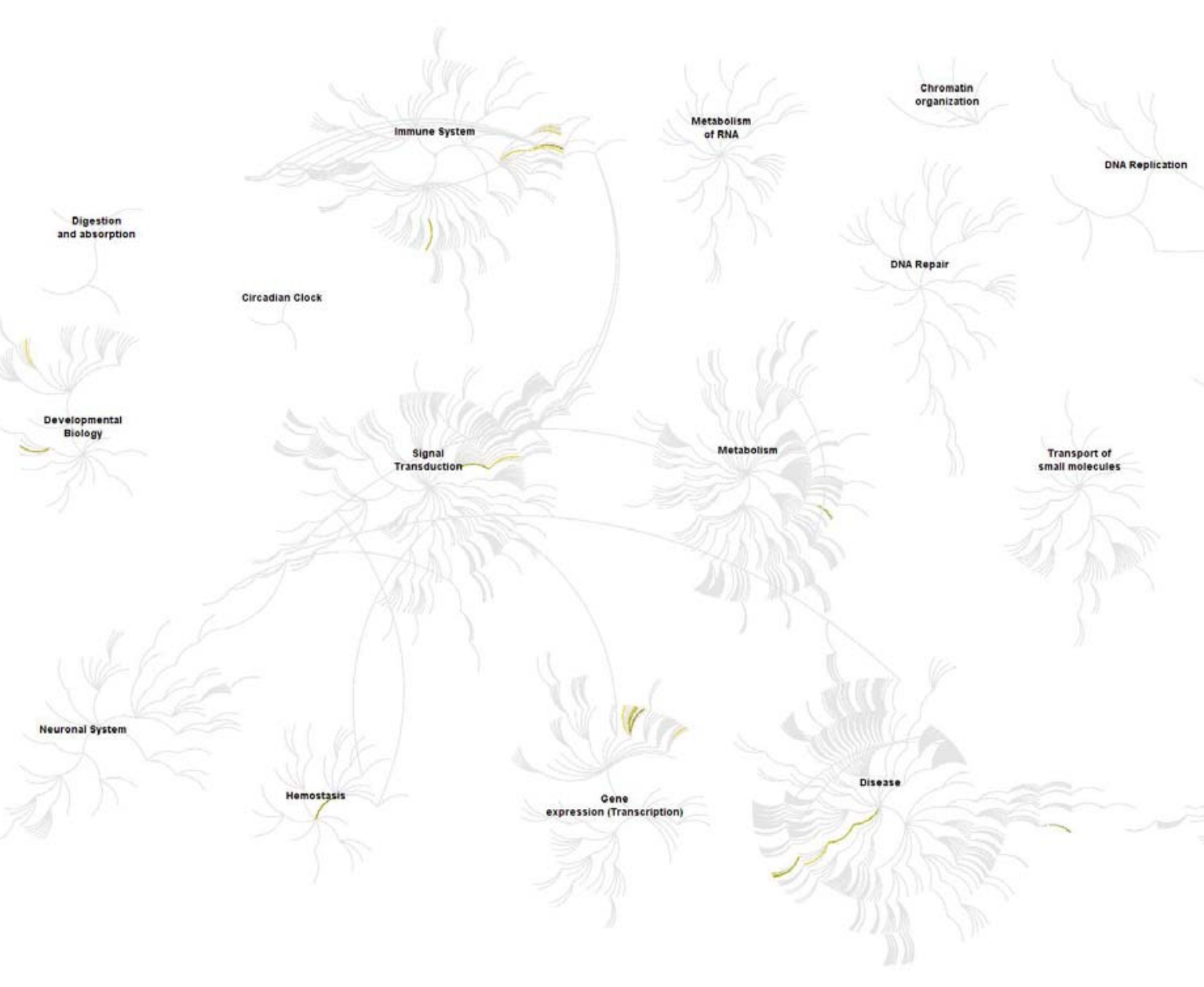

Figure 3. The Reactom pathway visualization. Input genes are represented by the top significantly changed genes obtained from the GSE153626 dataset $(P<0.01)$. The yellow color represents the most relevant signaling pathways. 


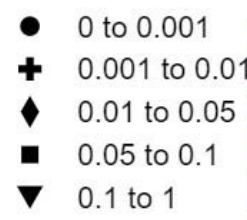

$\begin{array}{ll}-0 & \text { to } 0.001 \\ + & 0.001 \text { to } 0.01\end{array}$

0.01 to 0.05

$\boldsymbol{\nabla} 0.1$ to 1 protein synthesis inhibitor dopamine receptor antagonist NFkB pathway inhibitor topoisomerase inhibitor HDAC inhibitor ATPase inhibitor HMGCR inhibitor PI3K inhibitor tubulin polymerization inhibitor HSP inhibitor mTOR inhibitor adrenergic receptor antagonist MEK inhibitor

EGFR inhibitor

other

unknown

retinoid receptor agonist glucocorticoid receptor agonist CDK inhibitor adrenergic receptor agonist

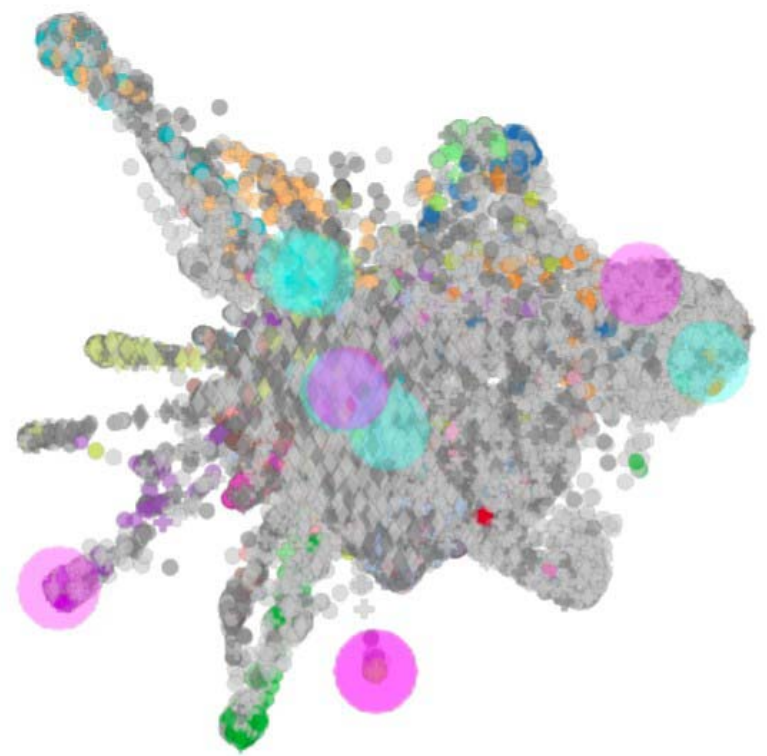

Figure 4. Inhibitor prediction against MM by L1000FDW visualization. Input genes are represented by the significantly changed genes obtained from the GSE153626 dataset. Dots are the Mode of Action (MOA) of the respective drug. 
Table 1

\begin{tabular}{llll}
\hline Entrez gene & Gene Symble & Fold-change & Regulation \\
\hline Top 10 down-regulated DEGs in COVID-19 & \\
222 & Aldh3b2 & -1.932183566 & Down \\
1800 & Dpep1 & -1.932183566 & Down \\
2529 & Fut7 & -1.932183566 & Down \\
100628598 & Mir5129 & -1.932183566 & Down \\
5319 & Pla2g1b & -1.932183566 & Down \\
100271841 & Uckl1os & -1.932183566 & Down \\
2334 & Aff2 & -1.928411441 & Down \\
201294 & Unc13d & -1.927922234 & Down \\
10924 & Smpdl3a & -1.926741966 & Down \\
54627 & Map10 & -1.925629606 & Down \\
Top 10 up-regulated DEGs in COVID-19 & \\
114987 & Wdr31 & 1.8938373435 & up \\
115487260 & LOC115487260 & 1.8192560767 & up \\
84254 & Camkk1 & 1.8173764446 & up \\
90407 & Tmem41a & 1.8149963395 & up \\
8624 & Psmg1 & 1.8132514217 & up \\
1807 & Dpys & 1.8100396352 & up \\
147015 & Dhrs13 & 1.8079677725 & up \\
4282 & Mif & 1.8068839504 & up \\
115485822 & LOC115485822 & 1.8050434401 & up \\
55810 & Gm16853 & 1.7967290544 & up \\
\hline
\end{tabular}


Table 2. Top ten genes demonstrated by connectivity degree in the PPI network

\begin{tabular}{lll}
\hline Gene Symbol & Gene title & Degree \\
\hline Gngt2 & G protein subunit gamma transducin 2 & 15 \\
Foxp3 & Forkhead box P3 & 12 \\
Cd3g & CD3g molecule & 10 \\
H2-Ab1 & Histocompatibility 2, class II antigen Ab1 & 9 \\
H2-Eb2 & Histocompatibility 2, class II antigen Eb2 & 8 \\
F2rl2 & Coagulation factor II thrombin receptor like 2 & 8 \\
Ccr5 & C-C motif chemokine receptor 5 & 7 \\
Ceacam1 & CEA cell adhesion molecule 1 & 7 \\
Vav3 & Vav guanine nucleotide exchange factor 3 & 7 \\
Lat & Linker for activation of T cells & 7 \\
\hline
\end{tabular}

